\title{
Asymmetric Tunneling Conductance in Doped Antiferromagnets
}

\author{
A. MACiA̧G* AND P. Wróbel \\ Institute for Low Temperature and Structure Research \\ P.O. Box 1410, 50-950 Wrocław 2, Poland
}

\begin{abstract}
We calculate the tunneling conductance in the framework of the spin polaron model, which is an effective model for the $t-J$ model, in the limit where at least short-range antiferromagnetic correlations exist. We show that both in the normal state and in the superconducting state the asymmetry of tunneling conductance is present. This fact is the implication of the particle-hole asymmetry of the spin polaron Hamiltonian in the limit of low density of spin polarons which are quasiparticles emerging in a hole doped antiferromagnet. Experimental evidence of analogous asymmetric tunneling conductance was found in the tunneling spectroscopy measurements of high $T_{\mathrm{c}}$ superconductors.
\end{abstract}

PACS numbers: 71.10.Fd, 74.20.Mn, 74.25.Fy

\section{Introduction}

Scanning tunneling microscope (STM) experiments have been one of the more important tools in the study of cuprates. One of the features of this experimental method is the fact, that it can measure density of states (DOS) near $E_{\mathrm{F}}$, and probe the states above and below $E_{\mathrm{F}}$. For that reason, STM is the proper method to study the electronic structure of cuprates. Recent papers on STM experiments done in $\mathrm{Bi}_{2} \mathrm{Sr}_{2} \mathrm{CaCu}_{2} \mathrm{O}_{8+\delta}$ [1] and other cuprates report that the tunneling conductivity is asymmetric as a function of voltage. When the doping decreases, the asymmetry becomes more visible. This phenomenon was analyzed by some other theoretical groups, but unfortunately these groups did not take into account the antiferromagnetic (AF) correlations in their calculations which influence the electronic structure of weakly doped cuprates. For example, the model used by Anderson and Ong [2] produces the Fermi surface (FS) different from the remnant FS, which has been observed [3] in cuprates. Randeria et al. [4] in their paper also neglect the role of antiferromagnetic correlations, and do not say much about the character of quasiparticle excitations. Thus, motivated by recent

${ }^{*}$ corresponding author; e-mail: a.maciag@int.pan.wroc.pl 
experiments we discuss the tunneling asymmetry in the framework of the spin polaron model (SPM), which is an effective model for the $t-J$ model $(t J \mathrm{M})$ on the square lattice. We assume that AF correlations, which are at least of short range, tend to confine motion of holes created in the AF spin background. The nature of quasiparticle propagation and interaction between quasiparticle is determined by a tendency to restore the local AF order. It was shown that the SPM describes many effects observed in weakly doped AFs [5, 6]. In this work, due to the lack of space, we will only sketch the theory of tunneling based on the Hamiltonian introduced by Cohen et al. [7]. In the framework of the SPM we will derive the conductance formula for the metal-metal (NIN) junction and the metal-superconductor (NIS) junction. The derivation of the spin polaron Hamiltonian on which our calculation relies on can be found in our previous paper [8].

\section{Tunneling Hamiltonian}

We write Hamiltonian in the form of three terms $H=H_{\mathrm{R}}+H_{\mathrm{L}}+H_{T}$, where $H_{\mathrm{R}}$ and $H_{\mathrm{L}}$ are the Hamiltonians for particles on the right and left side of the tunneling junction, and $H_{T}=\sum_{\boldsymbol{k} \boldsymbol{p}}\left(T_{\boldsymbol{k} \boldsymbol{p}} c_{\boldsymbol{k}}^{\dagger} c_{\boldsymbol{p}}+\right.$ h.c. $)$ is the term responsible for the tunneling. Hamiltonians $H_{\mathrm{R}}$ and $H_{\mathrm{L}}$ commute with each other and also term by term. They are expressed by means of operators $c_{k}^{\dagger}$ for the left side and $c_{\boldsymbol{p}}$ for the right side. $T_{\boldsymbol{k} \boldsymbol{p}}$ is the tunneling matrix element which we set to 1 in our approximation. The chemical potential on the left side of the junction $\mu_{\mathrm{L}}$ is different than the chemical potential on the right side $\mu_{\mathrm{R}}$. The difference is equal to applied voltage $e V=\mu_{\mathrm{L}}-\mu_{\mathrm{R}}$. The current may be expressed as the rate of change of the particle number. We will skip the detailed calculation, which can be found in Ref. [9]. The final formula for the total tunneling current is $I=-2 e \operatorname{Im}\left[\sum_{\boldsymbol{k} \boldsymbol{p}}\left|T_{\boldsymbol{k} \boldsymbol{p}}\right|^{2} \frac{1}{\beta} \sum_{i p} G_{\mathrm{L}}^{\text {ret }}(\boldsymbol{p}, i p) G_{\mathrm{R}}^{\text {ret }}(\boldsymbol{k}, i p-i \omega)\right]$. After integration over momentum $p$, the current flowing through NIN and NIS junction is respectively $I_{\mathrm{N}}=4 \pi e N_{\mathrm{L}} \sum_{\boldsymbol{k}}|T|^{2}\left[n_{\mathrm{F}}\left(\xi_{\boldsymbol{k}}\right)-n_{\mathrm{F}}\left(\xi_{\boldsymbol{k}}+e V\right)\right], I_{\mathrm{S}}=4 \pi e N_{\mathrm{L}} \sum_{\boldsymbol{k}}|T|^{2}\left\{v_{\boldsymbol{k}}^{2}[1-\right.$ $\left.\left.n_{\mathrm{F}}\left(E_{\boldsymbol{k}}\right)-n_{\mathrm{F}}\left(e V-E_{\boldsymbol{k}}\right)\right]+u_{\boldsymbol{k}}^{2}\left[n_{\mathrm{F}}\left(E_{\boldsymbol{k}}\right)-n_{\mathrm{F}}\left(e V+E_{\boldsymbol{k}}\right)\right]\right\}$. In the above formulae $N_{\mathrm{L}}$ is the density of states on the left side of the junction. Now, we can calculate the differential tunneling conductance: $\mathrm{d} I_{\mathrm{N}} / \mathrm{d} V=-4 \pi e N_{\mathrm{L}} \sum_{\boldsymbol{k}}|T|^{2} n_{\mathrm{F}}^{\prime}\left(\xi_{\boldsymbol{k}}+e V\right)$, $\mathrm{d} I_{\mathrm{S}} / \mathrm{d} V=-4 \pi e N_{\mathrm{L}} \sum_{\boldsymbol{k}}|T|^{2}\left[v_{\boldsymbol{k}}^{2} n_{\mathrm{F}}^{\prime}\left(e V-E_{\boldsymbol{k}}\right)+u_{\boldsymbol{k}}^{2} n_{\mathrm{F}}^{\prime}\left(e V+E_{\boldsymbol{k}}\right)\right]$, where $n_{\mathrm{F}}^{\prime}(E)=$ $\frac{\mathrm{d}}{\mathrm{d} T} n_{\mathrm{F}}(E)$. It should be stressed that we applied the spin polaron operators in the $H_{T}$ term for the right side of the junction, which is an approximation, because one could expect that bare holes participate in tunneling.

It is important to mention here that the low temperature conductance represents the density of states. Finally, we also introduce the quantity $c_{0}=4 \pi e N_{\mathrm{L}} C_{\mathrm{R}}$ which will be used later. $C_{\mathrm{R}}$ is the integral of $\mathrm{d} I_{\mathrm{N}} / \mathrm{d} V$ over energy.

\section{Numerical analysis and conclusion}

By using above formulae we obtain the differential tunneling conductance as a function of the applied voltage. All results were obtained for $5 \%$ of doping. 
Figure 1 depicts both the normalized conductance through NIN junction for the temperature $T=0.05 t$, where $t \sim 0.5 \mathrm{eV}$, when both sides of the junction are in the normal state, and the normalized conductance through the NIS junction for the temperature $T=0.005 t$, when the right side is in the SC state. In the latter case we use superconducting gap values obtained for the respective ground state calculated in the previous paper [8]. The asymmetry of DOS can be easily seen in Fig. 1. It is clear this phenomenon is related to the proximity of the Mott insulator state and to the presence of AF correlations. The main reason for the asymmetric behavior is the shape of the quasiparticle-energy dispersion which is presented in Fig. 2. It is clear that the band flattening near the point $(\pi, 0)$ is responsible for the asymmetric DOS.

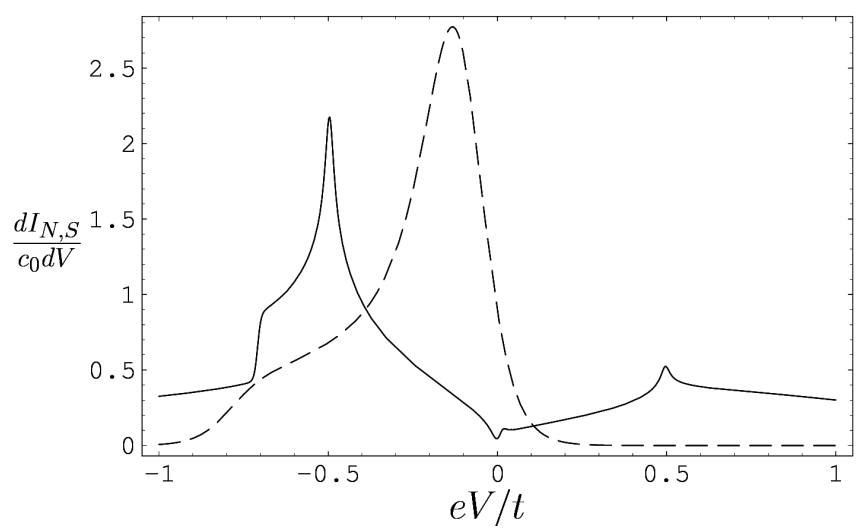

Fig. 1. Normalized differential conductance spectra through NIN junction calculated for $5 \%$ of doping and the temperature $T=0.05 t$ (dashed line), and through NIS junction calculated for $5 \%$ of doping and the temperature $T=0.005 t$ (solid line).

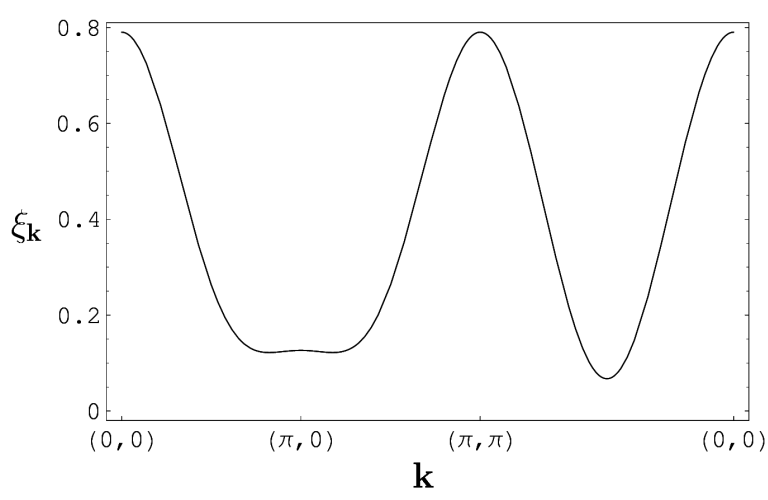

Fig. 2. Quasiparticle-energy dispersion.

In conclusion, our calculation shows that it is likely that AF correlations and the particle-hole asymmetry in the proximity to the Mott insulator phase are responsible for the tunneling current asymmetry observed in cuprates. 


\section{Acknowledgment}

P.W. acknowledges a partial support of the State Committee for Scientific Research (KBN) under contract No. 2P03B00925.

\section{References}

[1] Ch. Renner, B. Revaz, J.-Y. Genoud, K. Kadowaki, O. Fisher, Phys. Rev. Lett. 80, 149 (1998); Ch. Renner, O. Fisher, Phys. Rev. B 51, 9208 (1995).

[2] P.W. Anderson, N.P. Ong, cond-mat/0405518.

[3] F. Ronning, C. Kim, D.L. Feng, D.S. Marshall, A.G. Loeser, L.L. Miller, J.N. Eckstein, L. Bozovic, Z.-X. Shen, Science 282, 2067 (1998).

[4] M. Randeria, R. Sensarma, N. Trivedi, F.-C. Zhang, cond-mat/0412096.

[5] P. Wróbel, R. Eder, Phys. Rev. B 58, 15160 (1998).

[6] P. Wróbel, R. Eder, R. Micnas, J. Phys. Condens. Matter 15, 2755 (2003).

[7] M.H. Cohen, L.M. Falicov, J.C. Phillips, Phys. Rev. Lett. 8, 216 (1962).

[8] P. Wróbel, A. Maciąg, R. Eder, P. Fulde, R. Micnas, Acta Phys. Pol. A 106, 575 (2004).

[9] G.D. Mahan, in: Many-Particle Physic, Plenum Press, New York 1990, p. 788. 